ISSN 0103-9954

\title{
ESTIMATIVAS DAS PROPRIEDADES FÍSICO-MECÂNICAS DE COMPOSTOS LVL CONFECCIONADOS COM PARICÁ POR MEIO DE ULTRASSOM
}

\author{
ULTRASOUND NONDESTRUCTIVE METHOD TO PREDICT PHYSICAL-MECHANICAL \\ PROPERTIES OF LVL MADE FROM Schizolobium amazonicum
}

\author{
Rafael Rodolfo de Melo ${ }^{1}$ Cláudio Henrique Soares Del Menezzi²
}

\begin{abstract}
RESUMO
No presente estudo buscou-se avaliar as propriedades físicas e mecânicas de compostos LVL produzidos com a madeira de paricá (Schizolobium amazonicum Huber ex. Ducke) por meio do ultrassom. Para isso, foram utilizados LVL produzidos em laboratório e destes retiradas amostras para realização dos ensaios não destrutivos. Posteriormente, as mesmas amostras foram destinadas para realização dos ensaios físicos (absorção de água; inchamento em espessura e inchamento residual) ou mecânicos (resistência e rigidez à flexão estática flatwise; resistência e rigidez à flexão estática edgewise; resistência à compressão paralela e resistência ao cisalhamento paralelo e perpendicular). Os dados da velocidade de propagação de ondas e do módulo de elasticidade dinâmico obtidos nos ensaios não destrutivos foram utilizados no ajuste de equações para estimar as propriedades avaliadas. Os resultados obtidos apontaram o uso do ultrassom como uma ferramenta eficaz para a predição da maior parte das propriedades avaliadas. Dentre as variáveis independentes avaliadas, o módulo de elasticidade dinâmico obteve os melhores resultados quando comparado à velocidade de propagação das ondas.
\end{abstract}

Palavras-chave: ondas ultrassônicas; ensaios mecânicos; compostos estruturais de madeira.

\begin{abstract}
This study aimed at evaluating the utilization of ultrasound nondestructive method to estimate physical and mechanical properties of laminated veneer lumber (LVL) manufactured with Schizolobium amazonicum Huber ex. Ducke by ultrasound. For this, LVL boards were manufactured in laboratory. After manufacturing, the boards were nondestructively tested using ultrasound and afterwards the following physical and mechanical properties were assessed: water absorption; thickness swelling residual swelling, flatwise static bending; edgewise static bending; compression strength parallel to grain; shear strength parallel to glue-line; shear strength perpendicular to glue-line. Data about speed of sound and dynamic modulus of elasticity were used in different equations to estimate the properties evaluated. The results showed the use of ultrasound as an effective tool for prediction of most of the properties. Among the independent variables, the dynamic modulus of elasticity obtained the best results when compared to the speed of sound.
\end{abstract}

Keywords: ultrasonic waves; mechanical tests; structural composite lumber.

\section{INTRODUÇÃO}

Por meio das técnicas de avaliação não destrutiva é possível estimar as propriedades de materiais sem alterar a sua capacidade de uso final. Os princípios em que se baseiam os ensaios não destrutivos de madeira foram fundamentados por Jayne (1959), que propôs a hipótese de que as propriedades de conservação e dissipação de energia da madeira podem ser mensuradas e utilizadas como

1 Engenheiro Florestal, Dr., Professor Adjunto do Instituto de Ciências Agrárias e Ambientais, Universidade Federal de Mato Grosso, Av. Alexandre Ferronato, 1200, CEP 78557-267, Sinop (MT), Brasil. rrmelo@ufmt.br

2 Engenheiro Florestal, Dr., Professor Adjunto do Departamento de Engenharia Florestal, Universidade de Brasília, Campus Universitário Darcy Ribeiro, CEP 70904-970, Brasília (DF), Brasil. cmenezzi@unb.br 
mecanismos que determinam o comportamento esstático estrutural do material. Logo, podem ser estabelecidas relações matemáticas entre essas propriedades com a resistência e elasticidade da madeira e compostos de madeira.

Avaliações não destrutivas têm sido utilizadas para a verificação de peças estruturais de madeira ou derivados, classificando-as e permitindo uma melhor aplicação dessas. Estas técnicas possibilitam uma série de vantagens, sendo a principal delas a manutenção da integridade estrutural da madeira, ou seja, não sendo necessária a retirada de corpos de prova para a realização de ensaios em laboratório, na determinação das propriedades mecânicas (PELLERIN e ROSS, 2002; WANG et al., 2001). Oliveira e Sales (2002) destacam ainda que os métodos não destrutivos, em comparação com os métodos convencionais para caracterização da madeira, possibilitam uma maior rapidez na análise de um grande estoque de produtos e versatilidade para se adequar a rotinas padronizadas em linhas de produção.

Para Wang et al. (2001), no setor florestal o emprego de métodos não destrutivos contribui para uniformidade no desempenho de compostos estruturais de madeira. Por meio deles, é possível classificar estes produtos resultando em peças com características e desempenho bem definidos. Conforme mencionado por Derner et al. (2010), existem vários métodos que podem ser utilizados para avaliação não destrutiva. Dentre os mais utilizados podem ser citados - observação visual; observação auditiva; emissão de ondas de baixa frequência; método da deflexão; radiação Gama e Método do raio-X (DERNER et al., 2010). Dentre estes, a utilização de ondas acústicas tem sido o mais empregado, sendo o ultrassom o principal equipamento empregado para tal finalidade.

Calegari (2006) destaca que a utilização do ultrassom consiste na emissão de pulsos elétricos por um circuito eletrônico. Esses pulsos elétricos são conduzidos por cabos coaxiais e convertidos em ondas elásticas pelo cristal piezelétrico, localizado nos transdutores. As vibrações mecânicas deslocamse pelo material, que atenuam o sinal emitido pelo gerador. $\mathrm{O}$ sinal retardado é recuperado por outro cristal piezelétrico, sendo então amplificado e transformado em pulsos elétricos novamente para medir o tempo de propagação. Assim, tendo a distância e o tempo de percurso da onda, obtém-se sua velocidade.

Para Gonçalves \& Bartolomeu (2000), o uso do ultrassom pode ser útil não só na classificação mecânica da madeira e dos produtos de madeira, assim como para separar estes em classes de resistência. $\mathrm{O}$ processo executado nas máquinas classificadoras consiste na passagem das peças pelo equipamento, que sofrem uma deflexão conhecida ou recebem uma carga conhecida. Automaticamente, o equipamento faz a leitura da carga (no caso da deflexão aplicada) ou da deflexão (no caso da carga aplicada). De acordo com a faixa que caracteriza as classes de resistência, essas peças são marcadas.

$\mathrm{O}$ presente trabalho teve como objetivo estimar as propriedades físico-mecânicas de compostos LVL produzidos com paricá (Schizolobium amazonicum Huber ex. Ducke), utilizando a técnica de avaliação não destrutiva por meio de ultrassom.

\section{MATERIAL E MÉTODOS}

\section{Obtenção das lâminas e manufatura dos compostos LVL}

Foram utilizadas lâminas de paricá (Schizolobium amazonicum) de aproximadamente $0,271 \mathrm{~cm} \times 85 \mathrm{~cm} \times 115 \mathrm{~cm}$ em espessura, largura e comprimento, obtidas na empresa PORTIL - Portas Itinga Limitada ${ }^{\circledR}$, localizada no município de Dom Eliseu, estado do Pará. Lâminas isentas de defeitos foram selecionadas e levadas ao laboratório e redimensionadas em amostras de $0,271 \mathrm{~cm} \times 25 \mathrm{~cm}$ x $50 \mathrm{~cm}$. Antes da manufatura dos LVL as lâminas foram levadas à estufa durante 2 horas a temperatura de $50^{\circ} \mathrm{C}$, para redução do teor de umidade.

Para produção dos LVL foi empregado à resina fenolformaldeído (CR-7010) na gramatura de $200 \mathrm{~g} / \mathrm{m}^{2}$, formulado nas proporções 100:5:8:7 (resina; farinha de trigo; farinha de coco; e água). A farinha de trigo e a de coco foram usadas de acordo com a ficha técnica da resina fornecida pela empresa SIGroup Crios, fabricante do adesivo. A viscosidade observada para a mistura foi de $840 \mathrm{cP}$, medida a temperatura ambiente com um viscosímetro digital e o teor de sólidos foi de $59 \%$.

Após a aplicação manual do adesivo com auxílio de uma espátula foi realizada uma préprensagem a frio dos compostos LVL com auxílio de blocos de concretos durante 30 minutos. Em seguida, os LVL foram levados à prensa hidráulica e prensados por 12 minutos com pratos aquecidos a $140^{\circ} \mathrm{C}$ e pressão de $1,0 \mathrm{~N} / \mathrm{mm}^{2}$. Ao todo, foram produzidos 21 painéis com dimensões aproximadas 
de $25 \mathrm{~cm}$ x $2,2 \mathrm{~cm}$ x $50 \mathrm{~cm}$ (largura x espessura $\mathrm{x}$ comprimento). Ao término da prensagem, esperouse o desaquecimento destes que, em seguida, foram conduzidos à câmara climática com temperatura de $20 \pm 2^{\circ} \mathrm{C}$ e umidade relativa de $65 \pm 5 \%$.

\section{Realização dos ensaios não destrutivos}

Depois de climatizados, os LVL foram esquadrejados nas dimensões $24 \mathrm{~cm} \mathrm{x} 48 \mathrm{~cm}$ (largura x comprimento) e novamente conduzidos ao laboratório para serem tomadas suas massas e dimensões, as quais foram utilizadas no cálculo da massa específica. Na mesma ocasião foi determinada a velocidade de propagação de ondas $\left(V_{0}\right)$ obtida em um ponto central do painel utilizando o aparelho de ultrassom. Estes valores foram utilizados para o cálculo do módulo de elasticidade dinâmico $\left(E_{m d}\right)$ por meio das Equações 1 e 2 .

$$
\begin{gathered}
V_{0}=\frac{L}{t \times 10^{-6}} \\
C_{L L}=\frac{V_{0}^{2} \times \rho}{g} \times 10^{-5}
\end{gathered}
$$

(Equação 1)

(Equação 2)

Em que: $V_{0}=$ velocidade de propagação da onda, $\mathrm{m} / \mathrm{s} ; L$ = distância percorrida pela onda $\mathrm{m} ; t=$ tempo de trânsito da onda, $\mu \mathrm{s} ; C_{L L}=$ constante dinâmica obtida pelo ultrassom, $\mathrm{N} / \mathrm{mm}^{2} ; \rho=$ massa específica aparente, $\mathrm{kg} / \mathrm{m}^{3}$; $g=$ aceleração da gravidade, $9,804 \mathrm{~m} / \mathrm{s}^{2}$.

Em seguida, os painéis retornaram à marcenaria para retiradas das amostras destinadas à realização dos ensaios físicos e mecânicos. De cada composto produzido, foram obtidas 9 amostras com dimensões aproximadas de 2,2 $\mathrm{cm}$ x 2,2 $\mathrm{cm}$ x 40 $\mathrm{cm}$ (espessura $\mathrm{x}$ largura $\mathrm{x}$ comprimento) por meio de cortes longitudinais. Todas estas foram utilizadas para determinação da $V_{0}$ via e ultrassom.

Posteriormente, as amostras foram destinadas aos ensaios físicos - massa específica, teor de umidade, absorção de água, inchamento em espessura e inchamento residual; e mecânicas flexão estática, compressão e cisalhamento.

\section{Determinação das propriedades}

Antes da realização dos ensaios físicos e mecânicos as amostras foram levadas à câmara climática com temperaturas de $20 \pm 2^{\circ} \mathrm{C}$ e $65 \pm 5 \%$ de umidade relativa, permanecendo até atingirem massa constante.
$\mathrm{Na}$ avaliação das propriedades físicas dos LVL produzidos com paricá foram utilizadas recomendações da norma ASTM D 1037 (1999). Foram utilizadas 21 amostras de madeira por tratamento, nas dimensões $2,2 \mathrm{~cm}$ x $2,2 \mathrm{~cm}$ x $10 \mathrm{~cm}$. As dimensões e massa dos corpos de prova foram tomadas após a climatização. Em seguida, foram submersos em água durante 96 horas, quando tiveram suas dimensões e massas retomadas. Ao término do ensaio, as amostras foram novamente conduzidas à câmara climática e, após a estabilização, tiveram suas dimensão e massa obtidas novamente.

Os ensaios mecânicos foram conduzidos conforme recomendações da norma ASTM D 5456 (2006). Para o ensaio de resistência, a flexão foi avaliada nas posições flatwise e edgewise (Figura 1). Foram utilizadas 21 amostras/ tratamento/posição, nas dimensões de 2,2 cm x 2,2 $\mathrm{cm}$ x $40 \mathrm{~cm}$. A velocidade de aplicação de carga foi aproximadamente $2,5 \mathrm{~mm} / \mathrm{min}$ para um vão de 36 $\mathrm{cm}$ de comprimento. Na resistência à compressão, utilizaram-se 21 amostras por tratamento com dimensões de $2,2 \mathrm{~cm}$ x 2,2 cm x $10 \mathrm{~cm}$. As dimensões dos corpos de prova foram determinadas pela máxima relação comprimento/raio de giração (17 vezes) permitida pela norma. Com a velocidade do ensaio de $1 \mathrm{~mm} / \mathrm{min}$, determinou-se a resistência à compressão das amostras pela relação entre a força aplicada e a área sujeita ao esforço. Para o cisalhamento paralelo e perpendicular à linha de
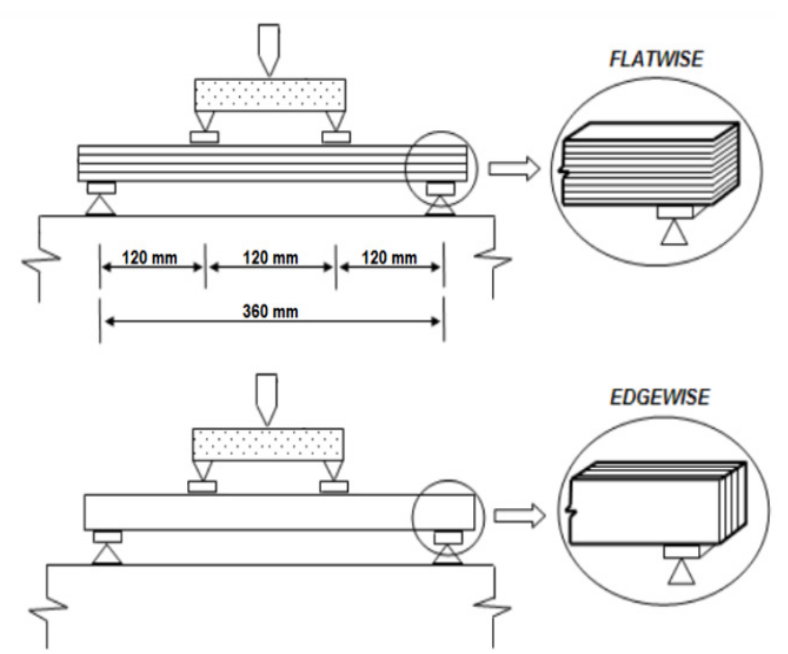

FIGURA 1: Modelo da realização dos ensaios de flexão estática nas posições flatwise e edgewise (Fonte: SOUZA, 2009).

FIGURE 1: Static bending schedule to evaluate flatwise and edgewise positions (Source: SOUZA, 2009). 
cola foram avaliadas 21 amostras com dimensões de 2,2 cm x 2,2 cm x 3,3 cm e uma área destinada à aplicação do esforço cisalhante.

\section{Análise dos resultados}

Para a análise dos resultados buscouse o ajuste de equações que visavam estimar o desempenho dos compostos LVL quanto as suas propriedades físicas (absorção de água; inchamento em espessura; e inchamento residual) e mecânicas (resistência e rigidez à flexão estática flatwise; resistência e rigidez à flexão estática edgewise; resistência à compressão paralela; e resistência ao cisalhamento paralelo e perpendicular) observadas nos ensaios não destrutivos, em função da velocidade de propagação das ondas e do módulo de elasticidade dinâmico obtidos pelo ultrassom. Adicionalmente, foi estimada a relação entre o $E_{m d}$ observado nos painéis e para a média das amostras retiradas de capa painel, obtidos pelas diferentes ferramentas de NDT.

\section{RESULTADOS E DISCUSSÕES}

Os valores da velocidade de propagação das ondas $\left(V_{0}\right)$ e do módulo de elasticidade dinâmico $\left(C_{L L}\right)$ obtidos por meio de ultrassom para os LVL confeccionados com a madeira de paricá podem ser observados na Tabela 1. Na Figura 2 podem ser observadas as relações entre o módulo de

TABELA 1: Valores da velocidade de propagação de ondas $\left(V_{0}\right)$ e do módulo de elasticidade dinâmico $\left(C_{L L}\right)$ obtidos por meio de ultrassom para os LVL de paricá utilizando.

TABLE 1: Values of the ultrasound velocity $\left(V_{0}\right)$ and dynamic modulus of elasticity $\left(C_{L L}\right)$ obtained by ultrasound for the LVL.

\begin{tabular}{ccc}
\hline Valores & $V_{0}(\mathrm{~m} / \mathrm{s})$ & $C_{L L}(\mathrm{MPa})$ \\
\hline Máximo & 5388 & 13347 \\
Médio & 5170 & 11762 \\
Mínimo & 4968 & 9952 \\
$\mathrm{CV}(\%)$ & 1,58 & 5,99 \\
\hline Total $^{1}$ & 198 & 198
\end{tabular}

Em que: ${ }^{1}$ número de amostras utilizadas para obtenção dos valores médios.

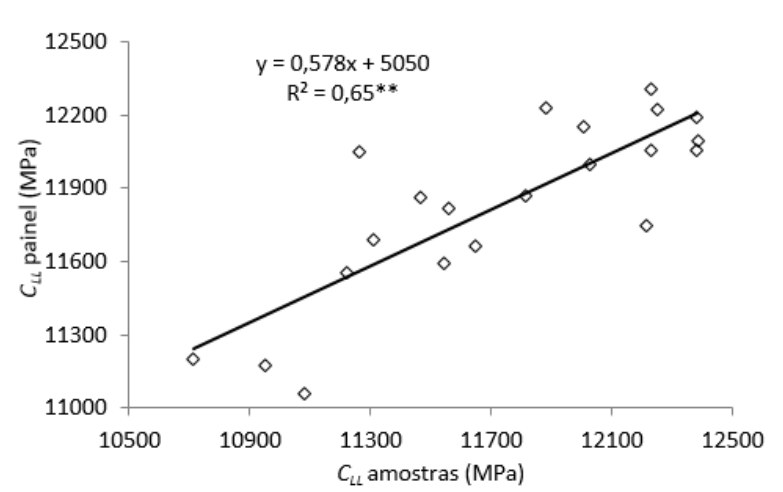

FIGURA 2: Relação entre o módulo de elasticidade dinâmico $\left(C_{L L}\right)$ observado nos compostos LVL e nas amostras utilizando o ultrassom.

FIGURE 2: Relationship between dynamic modulus of elasticity $\left(C_{L L}\right)$ observed in LVL boards and in samples.

elasticidade dinâmico $\left(C_{L L}\right)$ obtido para os LVL e os valores médios observados para os corpos de prova, utilizando o ultrassom. Apesar das equações geradas serem estatisticamente significativas em nível de $99 \%$ probabilidade, os valores observados para os coeficientes de determinação $\left(\mathrm{R}^{2}=0,63\right)$ foram menores que os observados na literatura, $\mathrm{o}$ que indica a variabilidade a longo da superfície de cada painel. Gabriel (2007), em análise semelhante, observou ajustes superiores a 0,80 .

\section{Propriedades Físicas}

A estimativa dos parâmetros físicos dos LVL de paricá utilizando os diferentes métodos de NDT podem ser observadas na Tabela 2. Embora diversas pesquisas venham demonstrado a eficácia dos NDT na estimativa das propriedades mecânicas de compostos LVL (MATOS, 1997; BORTOLETTO JÚNIOR, 2006; GABRIEL, 2007; ROSS et al., 2004; VALLANCE, 2009), poucos estudos têm sido desenvolvidos visando à utilização destas ferramentas na predição de parâmentos relacionados à estabilidade dimensional destes produtos. No presente estudo, foi possível estimar de forma significativa os percentuais de absorção de água e inchamento em espessura dos LVL.

Dentre as variáveis independentes avaliadas, o $C_{L L}$ apresentou a maior eficiência para a predição dos percentuais de absorção de água e inchamento em espessura (Figura 3). Não foi possível predizer de forma significativa o inchamento residual. 
TABELA 2: Modelos ajustados para predição das propriedades físicas dos compostos LVL em função da velocidade de propagação das ondas e do módulo de elasticidade dinâmico.

TABLE 2: Adjusted models to estimate physical properties of LVL as function of ultrasound velocity and dynamic modulus of elasticity .

\begin{tabular}{cccccc}
\hline$y$ & $x$ & Equação & $\mathrm{R}^{2}{ }_{\mathrm{aj}}$ & $\mathrm{S}_{\mathrm{yx}}$ & $\mathrm{F}$ \\
\hline \multirow{2}{*}{$\mathrm{AA}$} & $C_{L L}$ & $y=89,50-0,003 x$ & 0,58 & 2,03 & $84,86^{* *}$ \\
& $V_{0}$ & $y=-0,35+0,010 x$ & 0,06 & 3,04 & $4,12^{*}$ \\
\hline \multirow{2}{*}{$\mathrm{IE}$} & $C_{L L}$ & $y=-7,50+0,001^{*} x$ & 0,13 & 2,01 & $9,38^{* *}$ \\
& $V_{0}$ & $y=44,97-0,008^{*} x$ & 0,08 & 2,07 & $5,33^{*}$ \\
\hline \multirow{2}{*}{$\mathrm{IR}$} & $C_{L L}$ & - & - & - & $\mathrm{NS}$ \\
& $V_{0}$ & - & - & - & $\mathrm{NS}$ \\
\hline
\end{tabular}

Em que: $y=$ variável dependente; $x=$ variável independente; $\mathrm{AA}=$ absorção de água após 96 horas de imersão, em $\%$; IE = inchamento em espessura após 96 horas de imersão, em \%; IR = inchamento residual, em \%; $C_{L L}=$ constante dinâmica observado pelo ultrassom, em MPa; $V_{0}=$ velocidade de propagação das ondas, em m/s; ${ }^{\mathrm{Ns}}=$ não foram observadas correlações significativas em nível de $95 \%$ de probabilidade; ** e * significativo em nível de 1 e $5 \%$ erro.

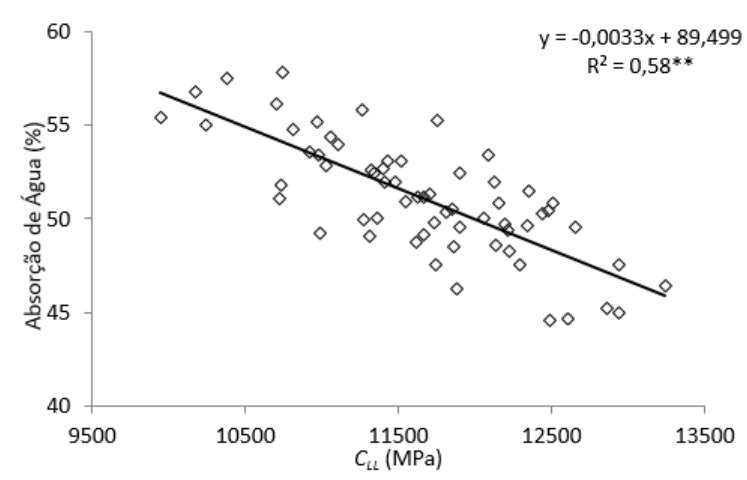

Esse resultado pode ser atríbuido, por ser este um parâmetro decorrente principalmente da liberação das tensões de compressão (DEL MENEZZI, 2006), tendo uma menor influência da composição física do material (propriedades morfológicas e anatômicas) que é o principal aspecto dignósticado pelos métodos não destrutivos (SHIMOYAMA, 2005).

De modo geral, na absorção de água, foi observada uma tendência de redução com o aumento do $C_{L L}$, enquanto no inchamento em espessura ocorreu o inverso. Esse comportamento pode ser atribuído ao fato de que em madeiras, ou compostos de madeiras, as variáveis $E_{m d} / C_{L L}$ são diretamente propocionais a sua massa específica (ROSS et al., 1998). Deste modo, conforme observado por Melo \& Del Menezzi (2010), uma maior absorção de água observada para as amostras com menor $C_{L L}$ pode ser atribuída ao maior volume de espaços vazios destas, que possam ser ocupados por água. Por outro lado, para o inchamento em espessura, amostras mais densas têm maior material e assim a contribuição do inchamento higroscópico é maior. Entretanto, Suchsland (1973) destaca que esta relação não é bem defenida, e que diferentemente do que ocorre para a madeira sólida, em que peças mais densas geralmente apresentam inchamento superior, no caso dos compostos de madeira, isto não ocorre necessariamente. of dynamic modulus of elasticity $\left(C_{L L}\right)$ obtained by ultrasound. 
TABELA 3: Modelos ajustados para predição resistência e rigidez em flexão dos LVL de paricá na posição flatwise em função da velocidade de propagação das ondas e do módulo de elasticidade dinâmico.

TABLE 3: Adjusted models for strength and stiffness static bending predicting of LVL in flatwise position depending on the speed of propagation of waves and elasticity dynamic modulus.

\begin{tabular}{rccccc}
\hline$y$ & $x$ & Equação & $\mathrm{R}_{\mathrm{aj}}^{2}$ & $\mathrm{~S}_{\mathrm{yx}}$ & $\mathrm{F}$ \\
\hline \multirow{2}{*}{$E_{m}$} & $C_{L L}$ & $y=-5.895,46+1,19 x$ & 0,48 & 944,98 & $57,42^{* *}$ \\
& $V_{0}$ & - & - & - & $\mathrm{NS}$ \\
$f_{m}$ & $C_{L L}$ & $y=-48,72+0,008 x$ & 0,45 & 6,61 & $50,78^{* *}$ \\
& $V_{0}$ & - & - & - & $\mathrm{NS}$ \\
\hline
\end{tabular}

Em que: $y=$ variável dependente; $x=$ variável independente; $E_{m d}=$ módulo de elasticidade em flexão, em MPa; $f_{m}=$ módulo de ruptura em flexão, em MPa; $C_{L L}=$ constante dinâmica observado pelo ultrassom, em MPa; $V_{0}=$ velocidade de propagação das ondas, em $\mathrm{m} / \mathrm{s}$; $\mathrm{NS}=$ não foram observadas correlações significativas em nível de $95 \%$ de probabilidade; ** e * = significativo em nível de 1 e $5 \%$ erro.

\section{Propriedades Mecânicas}

O ajuste de equações das amostras submetidas à flexão estática na posição flatwise, apontou que o $\mathrm{C}_{L L}$ pode ser utilizado na predição da resistência e rigidez dos LVL produzidos com paricá (Tabela 3). Resultados semelhantes foram observados por Xue \& Bu (2010) e Almeida (2011) ao avaliarem a utilização de diferentes métodos de avaliação não destrutiva. Já com uso da $V_{0}$ como variável independente não foi possível gerar modelos estatísticamente significativos para predição destes parâmetros.

$\mathrm{Na}$ comparação entre os ajustes das equações para o módulo de elasticidade $\left(E_{m}\right)$ e módulo de ruptura $\left(f_{m}\right)$ em flexão estática no sentido flatwise, foi observado para $E_{m}$ os melhores ajustes, independentemente do tipo do método utilizado (Figura 4). Segundo Stangerlin et al. (2011), esse comportamento se deve ao fato de que a tensão induzida durante os ensaios dinâmicos é pequena, ou seja, as medições dinâmicas são baseadas nas propriedades mecânicas apenas no limite elástico. O módulo de ruptura acontece em maior tensão e depois do limite elástico, resultando, consequentemente, em predições menos eficazes por meio dos ensaios não destrutivos. Grabiel (2007), Santos (2008) e Souza (2009) também observaram esse comportamento em LVL produzidos com lâminas de diferentes espécies florestais.

$\mathrm{Na}$ Tabela 4 são apresentados ajuste de modelos para estimativa do $E_{m} \mathrm{e} f_{m}$ em flexão estática na posição edgewise. Nos ensaios de flexão, assim como para as amostras submetidas aos esforços na posição flatwise, na posição edgewise foi possivel elaborar modelos com ajustes significativos utilizando o $C_{L L}$ para estimar os parâmetros $E_{m}$ e $f_{m}$

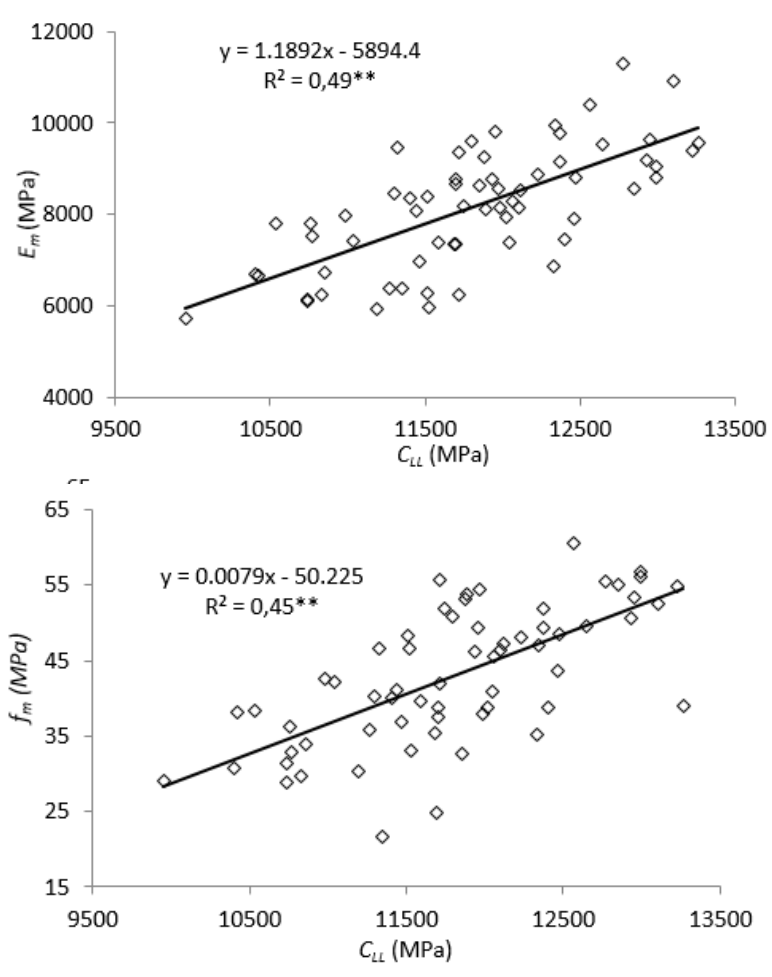

FIGURA 4: Estimativa dos módulos de elasticidade $\left(E_{m}\right)$ e ruptura $\left(f_{m}\right)$ na posição flatwise em função do módulo de elasticidade dinâmico $\left(C_{L L}\right)$ obtido por meio de ultrassom.

FIGURE 4: Estimates of modulus of elasticity $\left(E_{m}\right)$ and modulus of rupture $\left(f_{m}\right)$ in flatwise position depending on the dynamic modulus of elasticity $\left(C_{L L}\right)$ obtained by ultrasound. 
TABELA 4: Modelos ajustados para predição resistência e rigez dos LVL de paricá na posição edgewise em função da velocidade de propagação das ondas e do módulo de elasticidade dinâmico.

TABLE 4: Adjusted models for strength and stiffness static bending predicting of LVL in edgewise position depending on the speed of propagation of waves and dynamic modulus of elasticity.

\begin{tabular}{ccccccc}
\hline NDT & $y$ & $x$ & Equação & $\mathrm{R}_{\mathrm{aj}}^{2}$ & $\mathrm{~S}_{\mathrm{vx}}$ & $\mathrm{F}$ \\
\hline \multirow{3}{*}{$\mathrm{ULT}$} & $E_{m}$ & $C_{L L}$ & $y=-4.889,22+1,28 x$ & 0,39 & $1.043,35$ & $38,47^{* *}$ \\
& & $V_{0}$ & - & - & - & $\mathrm{NS}$ \\
& $f_{m}$ & $C_{L L}$ & $y=-19,56+0,006 x$ & 0,53 & 3,73 & $67,67^{* *}$ \\
& $V_{0}$ & - & - & - & $\mathrm{NS}$ \\
\hline
\end{tabular}

Em que: $y=$ variável dependente; $x$ = variável independente; $E_{m d}=$ módulo de elasticidade em flexão, em MPa; $f_{m}=$ módulo de ruptura em flexão, em MPa; $C_{L L}=$ constante dinâmica observado pelo ultrassom, em MPa; $V_{0}=$ velocidade de propagação das ondas, em m/s; ${ }^{\mathrm{Ns}}=$ não foram observadas correlações significativas em nível de $95 \%$ de probabilidade; $* *$ e $*$ significativo em nível de 1 e $5 \%$ erro.

(Figura 5). Também de forma similar ao observado na posição flawise, o uso da $V_{0}$ como variável independente não proporciou a criação
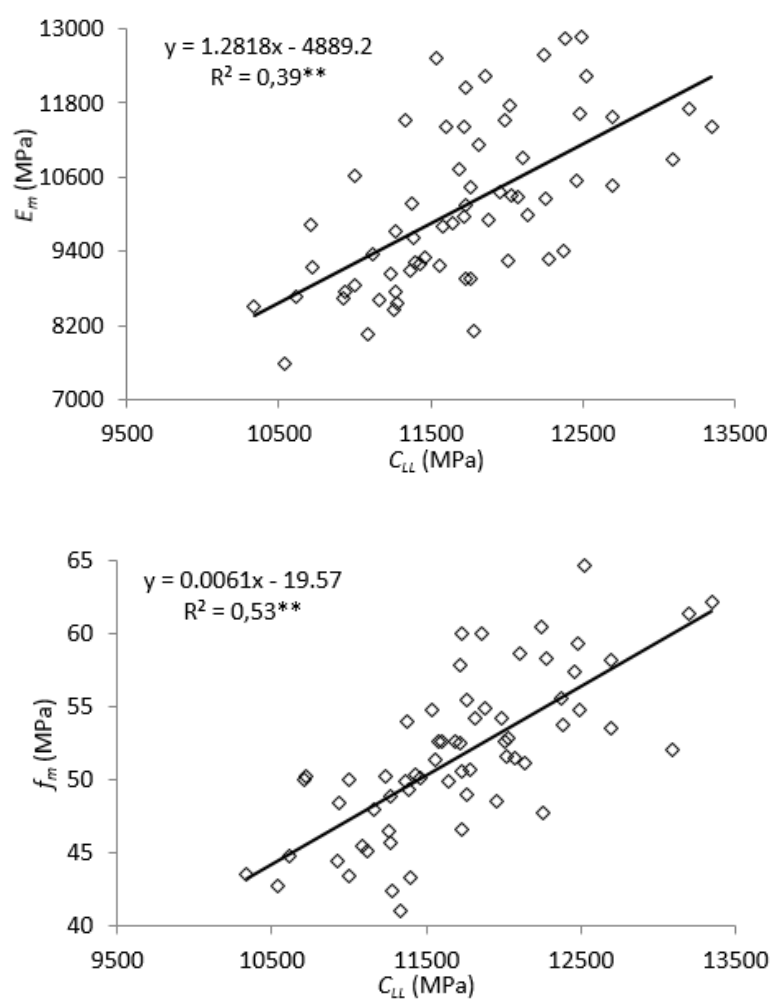

FIGURA 5: Estimativa dos módulos de elasticidade $\left(E_{m}\right)$ e ruptura $\left(f_{m}\right)$ na posição edwise em função do módulo de elasticidade dinâmico $\left(C_{L L}\right)$ obtido por meio de ultrassom.

FIGURE 5: Estimatives of modulus of elasticity $\left(E_{m}\right)$ and modulus of rupture $\left(f_{m}\right)$ in edgewise position depending on the dynamic modulus of elasticity $\left(C_{L L}\right)$ obtained by ultrasound. de modelos com ajuste de eficazes para predição dos módulos de elasticidade e ruptura, indicando ser a velocidade de propagação de ondas, isoladamente, inadequada para estimar as propriedades dos LVL produzidos com paricá em ensaios de flexão estática. Todavia, alguns autores como Halabe et al. (1997) e Souza et al. (2011) apontam a potencialidade de uso deste parâmetro na predição das propriedades de madeiras ou compostos de madeira.

Os resultados referentes à resistência à compressão paralela $\left(f_{c, 0}\right)$ e ao cisalhamento paralelo $\left(f_{v g, 0}\right)$ e perpendicular $\left(f_{v g, 90}\right)$ podem ser observados na Tabela 5. O uso do $C_{L L}$ possibilitou a geração de modelos satisfatórios para determinação da resistência a $f_{c, 0}$ (Figura 6). Este resultado está de acordo com o evidenciado por diversos autores (JUNG, 1982; CHA \& PEARSON, 1994; KRESTSCHMANN et al., 1993; KIMMEL \& JANOWIAK, 1995) que têm comprovado a eficiência dos métodos não destrutivos para determinação das constantes elásticas para compostos estruturais de madeira.

O ajuste de equação para estimar a resistência a $f_{v g, 0}$ e $f_{v g, 90}$ não foi possivel para a probabilidade mínima pré-estabelecida (95\%). Isso pode ser explicado pela variabilidade das amostras, já que foi muito pequena a dimensão das amostras ensaiadas destrutivamente, frente às que foram utilizadas nos ensaios não destrutivos. Especificamente, no caso do $f_{v g, 0}$, outro aspecto que pode ter contribuído para esse resultado é o fato de ser o adesivo o principal componente que fornece a resistência nesse tipo de ensaio. Neste sentido, para os fatores observados pelo ultrassom $\left(C_{L L}\right.$ e $\left.V_{0}\right)$, não é possível estimar características relacionadas à qualidade da ligação e, consequentemente, com o 
TABELA 5: Modelos ajustados para predição de resistência à compressão e ao cisalhamento paralelo ou perpendicular dos LVL de paricá em função da velocidade de propagação das ondas e do módulo de elasticidade dinâmico.

TABLE 5: Adjusted models for compression strength parallel, shear strength parallel and shear strength perpendicular depending on the ultrasound speed and dynamic modulus of elasticity.

\begin{tabular}{cccccc}
\hline$y$ & $x$ & Equação & $\mathrm{R}_{\text {aj. }}^{2}$ & $\mathrm{~S}_{\mathrm{yx}}$ & $\mathrm{F}$ \\
\hline \multirow{2}{*}{$f_{c, 0}$} & $C_{L L}$ & $y=-7,88-0,003 x$ & 0,36 & 2,85 & $34,20 * *$ \\
& $V_{0}$ & - & - & - & NS \\
\multirow{2}{*}{$f_{v g, 0}$} & $C_{L L}$ & - & - & - & NS \\
& $V_{0}$ & - & - & - & NS \\
\multirow{2}{*}{$f v g_{, 90}$} & $C_{L L}$ & - & - & - & NS \\
& $V_{0}$ & - & - & - & NS \\
\hline
\end{tabular}

Em que: $y=$ variável dependente; $x=$ variável independente; $f_{c, 0}=$ resistência à compressão, em N/ $\mathrm{mm}^{2} ; f_{v g, 0}=$ resistência ao cisalhamento paralelo à linha de cola, em N/mm $; f_{v g, 90}=$ resistência ao cisalhamento perpendicular, em N/mm $; C_{L L}=$ constante dinâmica observada pelo ultrassom, em MPa; $V_{0}=$ velocidade de propagação das ondas, em $\mathrm{m} / \mathrm{s}$; ${ }^{\mathrm{NS}}=$ não foram observadas correlações significativas em nível de $95 \%$ de probabilidade; ** e $*$ = significativo em nível de 1 e $5 \%$ erro.

emprego destes parâmetros, não foi possível gerar equações para predição da resistência neste tipo de ensaio (UYSAL, 2005).

No geral, o uso $V_{0}$ como variável independente não possibilitou o ajuste de modelos estatisticamente significativos para estimar as propriedades dos LVL produzidos com paricá. Embora o aumento da $V_{0}$ sofra influência direta da massa específica do madeira ou compostos de madeira (OLIVEIRA \& SALES,2000) e esta, por sua vez, está intimamente relacionada com a resistência destes materiais, diversos autores indicam que a velocidade de propagação das ondas é muito mais afetada pela estrutura anatômica da espécie do que por sua densidade (TEREZO, 2004; SHIMOYAMA, 2005). Tal aspecto faz com que, por exemplo, madeiras de coníferas que são normalmente menos densas, apresentam, em geral, velocidades maiores do que as dicotiledôneas. Isto ocorre porque as coníferas, em sua maioria, apresentam estrutura mais homogênea e um maior

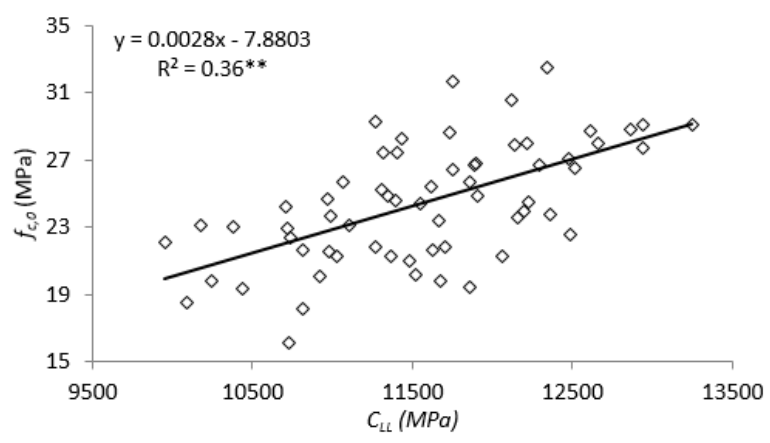

FIGURA 6: Estimativa da resistência à compressão paralela às fibras $\left(f_{c, 0}\right)$ em função do módulo de elasticidade dinâmico $\left(C_{L L}\right)$ obtido por meio de ultrassom.

FIGURE 6: Estimatives of compression strength parallel $\left(f_{c, 0}\right)$ depending on the dynamic modulus of elasticity $\left(C_{L L}\right)$ obtained by ultrasound.

comprimento dos fibrotraqueoides, o que, por sua vez possibilita maior velocidade na propagação das ondas (BUCUR, 2006).

\section{CONCLUSÕES}

O método de avaliação não destrutiva por ultrassom pode ser utilizado na predição da maior parte das propriedades físicas e mecânicas dos LVL produzidos com paricá. Exceções foram observadas para o inchamento residual e a resistência a esforços de cisalhamento paralelo ou perpendicular, em que o uso do ultrassom não possibilitou ajustes de modelos significativos. O módulo de elasticidade dinâmico foi o parâmetro que melhor adequou-se para estimar as propriedades dos LVL. Já a velocidade de propagação das ondas, na maioria dos casos, não permitiu o ajuste de modelos estatisticamente significativos.

\section{REFERÊNCIAS BIBLIOGRÁFICAS}

ALMEIDA, C.F.A.M. Uso de avaliação não destrutiva em vigotas de angelim vermelho. 43f. 2011. Monografia (Graduação em Engenharia Florestal) - Universidade de Brasília, Brasília, 2011. AMERICAN SOCIETY FOR TESTING AND MATERIALS - ASTM D 1037. Standard test methods of evaluating properties of wood-based fiber and particle panel materials. ASTM D 1037.

Annual book of ASTM standards, 1999. 
AMERICAN SOCIETY FOR TESTING AND MATERIALS - ASTM D 5456. Standard specification for evaluation of structural composite lumber products. Annual book of ASTM standards, 2006.

BODIG, J. The process of NDE research for wood and wood composites. The e-Journal of Nondestructive Testing, v.26, n.3, 2001.

BODIG, J.; JAYNE, B.A. Mechanics of wood and wood composites. Van Nostrand Reinhold, New York, 736p. 1993.

BORTOLETTO JÚNIOR, G. Produção de lâminas, compensado e laminated veneer lumber - LVL a partir da madeira de Pinus merkusii. 110f. 2006. Tese (Livre Docência) - Universidade de São Paulo/ ESALQ - Piracicaba, 2006.

BUCUR, V. Acoustics of wood. $2^{\mathrm{a}}$ ed. Basiléia: Birkhäuser. 2006. 393p.

CALEGARI, L. Uso da onda ultra-sônica como meio de controle do processo de secagem da madeira. Santa Maria: UFSM, 2006. 91f. Dissertação (Mestrado em Engenharia Florestal) Universidade Federal de Santa Maria, 2006.

CARVALHO,A.M.;LAHR, F.A.R.;BORTOLETTO JÚNIOR, G. Use of Brazilian eucalyptus to produce LVL panels. Forest Products Journal, Peachtree Corners, v.54, n.11, p.61-64, 2004.

CHA, J.K.; PEARSON, R.G. Stress analysis and prediction in 3-layer laminated veneer lumber: response to crack and grain angle. Wood and Fiber Science, v.26, n.1, p.97-106, 1994.

DEL MENEZZI, C.H.S. Instabilidade dimensional da madeira e de seus subprodutos: causas, conseqüências e prevenção. Brasília-DF: UnB, 2006. 37p. (Comunicações Técnicas Florestais, v.8, n.2).

DERNER, L.A.; TEREZO, R.T.; SZÜCS, C.A. Avaliação do módulo de elasticidade da espécie paricá através de ensaio não destrutivo. In: ENCONTRO BRASILEIRO EM MADEIRA E ESTRUTURAS DE MADEIRA, 10, 2010. Londrina. Anais... Londrina: UEL, 2010.

ERIKSON, R.G. et al. Mechanical grading of lumber sawn from small-diameter lodge pole pine, ponderosa pine and grand fir trees from northern Idaho. Forest Products Journal, Peachtree Corners, Peachtree Corners, v.50, n.7/8, p.59-65. 2000.

GABRIEL, M.S.C. Desempenho físico-mecânico de painéis $L V L$ de pinus tropicais da região de São Paulo. Botucatu 2007, 125f. Tese (Doutorado em Agronomia - Energia na Agricultura) - Faculdade de Ciências Agronômicas, Universidade Estadual Paulista - UNESP.

GONÇALVES, R.; BARTHOLOMEU, A. Avaliação do desempenho de ensaio não destrutivo em vigas de madeira de Eucalyptus citriodora e Pinus elliottii. Revista Brasileira de Engenharia Agrícola e Ambiental, v. 4, n. 2, p. 269-274, fev. 2000.

HALABE, U.B. et al. Nondestructive evaluation of green wood using stress wave and transverse vibration techniques. Materials Evaluation, v.55, n.9, p.1013-1018, 1997.

HAN, G.; WU, Q.; WANG, X. Stress-wave velocity of wood based panels: effect of moisture, product type, and material direction. Forest Products Journal, Peachtree Corners, v.56, n.1, p. 28-33, 2006.

JAYNE, B.A. Vibrational properties of wood as indices of quality. Forest Products Journal, Madison, Peachtree Corners, v.9, n.11, p.413-416, 1959.

JUNG, J. Properties of parallel laminated veneer from stress-wave-tested veneers. Forest Products Journal, Peachtree Corners, Madison, v.32, n.7, p.30-35, 1982.

KIMMEL, J.D.; JANOWIAK, J.J. Red maple and yellow-poplar LVL from ultrasonically rated veneer. Forest Products Journal, Peachtree Corners, v.45, n. 7/8, p.54-58, 1995.

KRETSCHMANN, D.E. et al. Effect of various proportions of juvenile wood on laminated veneer lumber. USDA. Forest Service. Research Paper, FPL-521, Madison, p1-31, 1993.

MATOS, J.L.M. Estudo sobre a produção de painéis estruturais de lâminas paralelas de Pinus taeda L. Tese (Doutorado em Ciências Florestais). Universidade Federal do Paraná, Curitiba 1997. $117 \mathrm{p}$.

MELO, R.R.; DEL MENEZZI, C.H.S. Influência da massa específica nas propriedades físico-mecânicas de painéis aglomerados. Silva Lusitana, v.18, n.1, p.59-73, 2010.

MORALES, E.A.M. Técnicas de propagação de ondas na estimativa de propriedades mecânicas de painéis OSB. 2006, 94f. Tese (Doutorado em Ciência e Engenharia de Materiais) - Universidade de São Paulo, São Carlos, 2006.

NOGUEIRA, M.; BALLARIN, A.W. Efeito da idade na qualificação mecânica da madeira serrada de Eucalyptus sp. com uso de ensaios não-destrutivos. Revista Energia na Agricultura, v.23, n.3, p.74-94, 2008. 
OLIVEIRA， F.G.R.; SALES， A. Ultrasonic measurements in Brazilian hardwood. Materials Research, v. 5, n. 1, p. 51-55. 2002.

PELLERIN, R.F.; ROSS, J.R. Nondestructive evaluation of wood. Madison: FPS. 2002, 210p.

ROSS, R.J. et al. Yield and ultrasonic modulus of elasticity of red maple veneer. Forest Products Journal, Peachtree Corners, v.54, n.12, p.220-225, 2004.

SANTOS,A.M.L. Avaliação teórica experimental de vigas em "I" pré-fabricadas de madeira com flange de painéis de lâminas paralelas (LVL) e alma de painéis de partículas orientadas (OSB) e compensado. 2008, 94f. Dissertação (Mestrado em Ciências Florestais) - Universidade de Brasília, Brasília, 2008.

SHIMOYAMA, V.R.S. Estimativas de propriedades da madeira de Pinus taeda através do método não-destrutivo de emissão de ondas de tensão, visando à geração de produtos de alto valor agregado. Curitiba: Universidade Federal do Paraná, 2005. 151p. Tese de Doutorado.

SOUZA, F. Propriedades mecânicas, físicas, biológicas e avaliação não destrutiva de painéis de lâminas paralelas (LVL) Confeccionados com Madeira de Pinus oocarpa e Pinus kesiya. 2009. 135f. Dissertação (Mestrado em Ciências Florestais) - Universidade de Brasília, Brasília, 2009.

SOUZA, F.; DEL MENEZZI, C.H.S.; BORTOLETTO JÚNIOR, G. Material properties and nondestructive evaluation of laminated veneer lumber (LVL) made from Pinus oocarpa and P. kesiya. European Journal of Wood and Wood Products, v.69, 183-192, 2011.

SOUZA, F. et al. Influência da espécie e do eixo estrutural na velocidade de propagação de ondas de tensão em painéis de lâminas paralelas (LVL). In: $5^{\circ}$ SIMPÓSIO DE PÓS-GRADUAÇÃO EM
CIÊNCIAS FLORESTAIS, Brasília DF. Anais... v.1, p409-419, 2008.

STANGERLIN, D.M.; CADEMARTORI, P.H.G.; GATTO, D.A.; CALEGARI, L.; MELO, R.R.; VIVIAN, M.A.; MODES, K.S. Propagação indireta e semidireta de ondas ultrassonoras na estimativa de propriedades mecânicas da madeira. Ciência da Madeira, v.02, n.02, p.85-95, 2011.

SUCHSLAND, O. Hygroscopic thickness swelling and related properties of selected commercial particleboards. Forest Products Journal, Peachtree Corners, v.23, n.7, p.26-30, 1973.

TEREZO, R.F. Propriedades mecânicas de madeiras utilizadas em estruturas históricas e contemporâneas estimadas por meio de ultrasom. 119f. 2004. Dissertação (Mestrado em Engenharia Civil) - Universidade Federal de Santa Catarina, Florianópolis, 2004.

UYSAL, B. Bonding strength and dimensional stability of laminated veneer lumbers manufactured by using different adhesives after the steam test. International Journaul od Adhesion and Adhesives, v.25, n.5, p.395-403, 2005.

VALLANCE, D.B. Non-destructive evaluation of veneer using optical scanning and ultrasonic stress wave analysis systems. 2009, 279f. Tese (Doutorado em "Wood Science") - Oregon State University, 2009.

WANG, X.; CARTER, P.; ROSS, R.J.; BRASHAW. Acoustic assessment of wood quality of raw forest materials - a path to increased profitability. Forest Products Journal, Peachtree Corners, Madison, v.57, n.5, p.6-14, 2007.

XUE, B.; HU, Y. Nondestructive testing of mechanical properties of pine laminated veneer lumber. Advanced Materiasl Research, v.150/151, n.10, p.1435-1428, 2011. 